\title{
Fine Particulate Matter Exposure and Blood Pressure: Evidence from a Chinese Large Multiple Follow-Up Study
}

\section{Bahabaike Jiangtulu}

Peking University

Changxin Lan

Peking University

Junxi Chen

Peking University

Bin Wang ( $\square$ binwangpku@foxmail.com )

Peking University https://orcid.org/0000-0002-1164-8430

Tao Xue

Peking University

\section{Research Article}

Keywords: Fine particulate matter, PM2.5, blood pressure, risk

Posted Date: November 23rd, 2021

DOI: https://doi.org/10.21203/rs.3.rs-1067685/v1

License: (c) (1) This work is licensed under a Creative Commons Attribution 4.0 International License. Read Full License 


\section{Abstract \\ Background:}

Long-term exposure to fine particulate matter ( $\mathrm{PM}_{2.5}$ or FPM) may cause adverse effects on cardiovascular diseases. However, evidence that whether improved air quality can decrease blood pressure (BP) in humans is still needed from a large population study.

\section{Methods:}

Our study aimed to investigate the association of population ambient $\mathrm{PM}_{2.5}$ exposure with the blood pressure (BP) changes in China with implementing the Action Plan on Air Pollution Prevention and Control. A total of 14,080 participants who had at least two valid visits were adopted from the China Health and Retirement Longitudinal Survey (CHARLS) during 2011-2015. Their long-term $\mathrm{PM}_{2.5}$ exposure was assessed at the geographical level of a regular $0.1^{\circ} \times 0.1^{\circ}$ grid over China. A mixed-effects regression model was used to assess their associations. The robustness and homogeneity of the association were tested via sensitivity analyses.

\section{Results:}

The results revealed that each reduction of $10 \mu \mathrm{g} / \mathrm{m}^{3}$ in the 1 year-mean $\mathrm{PM}_{2.5}$ concentration $\left(F P M_{1 Y}\right)$ was associated with a decrease of 1.24 (95\% confidence interval [Cl]: $0.84-1.64) \mathrm{mmHg}$ of systolic BP (SBP) and 0.50 (95\% Cl: $0.25-0.75) \mathrm{mmHg}$ of diastolic BP (DBP), respectively. A robust association was observed between the long-term reduction of $\mathrm{PM}_{2.5}$ and decreased $\mathrm{BP}$ in the middle-aged and elderly population in China. These findings were further confirmed by a non-linear regression model.

\section{Conclusions:}

We concluded that air pollution control for $\mathrm{PM}_{2.5}$ can obviously promote vascular health. Our study provided robust scientific support for making the air pollution control policies.

\section{Key Messages}

- A robust association was observed between the long-term reduction of $\mathrm{PM}_{2.5}$ and decreased BP in the middle-aged and elderly population in China.

- Nonlinear regression verified that the decreasing trend in BP with decreases in $\mathrm{PM}_{2.5}$ was almost linear. 
- The Mean (IQR) $\mathrm{PM}_{2.5}$ was decreased significantly from 61.3 (27.6) to $52.8(24.0) \mu \mathrm{g} / \mathrm{m}^{3}$ during 2011-2015.

- Air pollution control for $\mathrm{PM}_{2.5}$ can obviously promote vascular health.

\section{Introduction}

High blood pressure, also known as hypertension, is blood pressure that is consistently higher than what is considered normal. There are 2 types of blood pressure measures: systolic and diastolic. Systolic blood pressure is the pressure in the arteries when the heart beats, while diastolic pressure is the pressure in the arteries when the heart rests. Normal systolic blood pressure is less than 120 millimeters of mercury $(\mathrm{mmHg})$, and normal diastolic blood pressure is less than $80 \mathrm{mmHg}$, together described as 120/80 $\mathrm{mmHg}$ (Desai et al., 2020). Hypertension has been well recognized as a major risk factor for cardiovascular diseases (CVDs) (Forouzanfar et al., 2017). Hypertension is also a leading risk factor of mortality and disability globally (Stanaway et al., 2018). In China, the prevalence rate of hypertension among adults over 35 years of age is $32.5 \%$, resulting in various adverse health outcomes and a heavy financial burden (Lewington et al., 2016). The prevalence of hypertension in Chinese adults has been increasing (Wang et al., 2018; Bao and Wang, 2020; Ma et al., 2021). Previous studies reported that highdensity lipoprotein cholesterol, triglycerides, body mass index, alcohol dependence, insomnia, educational level, diabetes, smoking, stress, viral infection, and age are risk factors for developing hypertension (Hay et al., 2020; van Oort et al., 2020). It also has been reported that population blood pressure (BP) can be affected by environmental factors and their interactions with genetics (Bochud and Guessous, 2012). Among them, the effects of air pollution on hypertension have been extensively studied (Huang et al., 2019; Zhang et al., 2019b). Numerous studies have indicated that long-term fine particulate matter $\left(\mathrm{PM}_{2.5}\right.$ or FPM) exposure increases the risk of hypertension (Lin et al., 2017; Liu et al., 2017; Xie et al., 2018a; Wu et al., 2020). Both human and animal experimental studies have provided several pathways to partially explain the association between $\mathrm{PM}_{2.5}$ exposure and the increased BP. The detailed mechanism behind the association still remains unclear. Previous studies show $\mathrm{PM}_{2.5}$ can interact with vascular endothelium and cause oxidative stress and endothelium damage by circulating toxic components. Fewer inhalation of particulate matter might reduce the redox alterations in the vessel wall and in turn decrease aortic vascular tone (Rao et al., 2018). However, few studies have assessed whether blood pressure is decreased by a cleaner ambient environment. The Chinese population has been exposed to air that is severely polluted by $\mathrm{PM}_{2.5}$ since the 2000s (Xue et al., 2019b). In 2013, the well-known Air Pollution Prevention and Control Action Plan (APPCAP) was released by the Chinese government as the first national strategy on air pollution control (The State Council, 2013), which has markedly improved the air quality (Wang et al., 2019a). However, there is a lack of evidence to assess whether BP can be reduced by improved ambient air quality.

The association between ambient $\mathrm{PM}_{2.5}$ exposure and $\mathrm{BP}$ has been reported by various epidemiological studies (Xie et al., 2018b; Yang et al., 2018; Santos et al., 2019; Zhang et al., 2019b). Those studies tended to support that high exposure to ambient $\mathrm{PM}_{2.5}$ is significantly associated with an elevated $\mathrm{BP}$ over the 
short term (Dvonch et al., 2009; Dai et al., 2016). However, epidemiological evidence on the long-term effects of ambient $\mathrm{PM}_{2.5}$ on $\mathrm{BP}$ is inconsistent. Some studies have found significant associations between long-term $\mathrm{PM}_{2.5}$ exposure and increased BP (Chuang et al., 2011; Fuks et al., 2017; Zhang et al., 2018). For example, a cross-sectional study reported that each $10 \mu \mathrm{g} / \mathrm{m}^{3}$ increment in the 2-year average $\mathrm{PM}_{2.5}$ concentration was associated with increases of $0.45 \mathrm{mmHg}(95 \% \mathrm{Cl}: 0.40-0.50)$, and $0.07 \mathrm{mmHg}$ (95\% Cl: 0.04-0.11), in SBP, and DBP respectively (Zhang et al., 2018). Chan et al. found that long-term $\mathrm{PM}_{2.5}$ and nitrogen dioxide $\left(\mathrm{NO}_{2}\right)$ exposures were associated with higher blood pressure in the Sister Study (Chan et al., 2015b). However, some other study did not find their positive associations (Auchincloss et al., 2008; Bilenko et al., 2015; Chen et al., 2015b; Chung et al., 2015; Curto et al., 2019). For example, Chung et al. analyzed cross-sectional data from 27,752 Taipei City residents $>65$ years of age and reported that 1-year average $\mathrm{PM}_{2.5}$ was not associated with BP (Chung et al., 2015). It may due to the weak effect of $\mathrm{PM}_{2.5}$ exposure on the BP changes in a short period with low $\mathrm{PM}_{2.5}$ concentration. Compared with Taipei City, the concentration of $\mathrm{PM}_{2.5}$ in other cities in China is higher and $\mathrm{PM}_{2.5}$ dominate air pollutant concentrations in most cities in China. Therefore, long-term follow-up studies are needed to provide more valuable evidence about the casual relationship between $\mathrm{PM}_{2.5}$ exposure and the change in population BP.

A significant decrease in $\mathrm{PM}_{2.5}$ concentration was reported nationwide because of the introduction of a series of clean air policies in China from 2013 to 2017, especially Action Plan on Air Pollution Prevention and Control (APPCAP) issued by the China State Council (Huang et al., 2018a). The APPCAP focused in particular on three key regions, i.e., the Beijing-Tianjin-Hebei (BTH) area, the Yangtze River Delta (YRD), and the Pearl River Delta (PRD). Ten key actions and 35 concrete measures were put forward covering various aspects of air quality management, including the upgrading of industrial structure, adjustment of energy structure, point and non-point source pollution control, and management mechanisms and safeguard measures (The State Council, 2013). On the whole, APPCAP emphasized control multiple pollutants including $\mathrm{SO}_{2}, \mathrm{NO}_{x}$, primary particulate matter and the economic structural adjustment, energy cleanliness, and comprehensive control of multiple pollution sources (Feng et al., 2019). The populationweighted annual average $\mathrm{PM}_{2.5}$ concentration decreased from 61.8 (95\% Cl: 53.3-70.0) to 42.0 (95\% Cl: 35.7-48.6) $\mathrm{\mu g} / \mathrm{m}^{3}$ over the 5-year period (Zhang et al., 2019a). Since 2013, China has conducted a series of air pollution prevention and control policies to improve air quality, which has created an observational quasi-experimental scenario to assess whether improving air quality is associated with a decrease in BP (Xue et al., 2019a). Therefore, identifying the relationship between long-term $\mathrm{PM}_{2.5}$ exposure and BP would provide a policy-making reference for other countries to balance economic development with human health. We hypothesized that a reduction in the ambient $\mathrm{PM}_{2.5}$ concentration would be associated with a decrease in BP. Herein, we performed a quasi-experimental study of the relationship between reduction in $\mathrm{PM}_{2.5}$ and changes in $\mathrm{BP}$ based on a national survey conducted before and after the clean air policies in 2011 and 2015.

\section{Methods}




\section{Population Recruitment}

We obtained population health data from the China Health and Retirement Longitudinal Survey (CHARLS), which is publicly available at http://opendata.pku.edu.cn. The details of this project have been documented previously (Zhao et al., 2014). Briefly, to ensure the national representativeness of the project, the study population was selected from 28 provinces (150 counties or districts) via multistage probability sampling in China (Figure S1). Face-to-face interviews were performed every 2 years using a standard questionnaire to collect basic information on socio-demographics (home address, age, gender, and educational level), energy-use characteristics for cooking and heating, health-related behaviors (smoking and drinking), and health status (self-reported general health and medicine usage).

Standardized resting BP measurements were performed by trained nurses. Left upper-arm BP was measured three times in the sitting position at 45-second intervals, and the mean value was recorded. Currently available are the CHARLS waves for 2011, 2013, and 2015, which cover the periods before and after the clean air actions in China. The national baseline survey individual response rate was $80.5 \%$. Of the $19.5 \%$ rate of nonresponse, $8.8 \%$ was due to refusal to respond, $8.2 \%$ to the inability of interviewers to contact sample residents, and $2.0 \%$ to other reasons (Zhao et al., 2014). The surveillance results had a very high response rate and quality, which have been widely recognized in academia (Khera et al., 2018; Minicuci et al., 2019). We selected the most recently published data from the CHARLS. CHARLS is a nationally representative longitudinal survey of persons in China aged 40 years or older and their spouses[http://charls.pku.edu.cn/en]. Variables including gender, age, region, residence, education, smoking and drinking history, marital status, disease history, cooking energy, indoor temperature, antihypertension drugs (medication) were extracted from the questionnaire, and utilized as covariates to control for confounders. Missing data in covariates included in our study were imputed by multiple imputation method. In our study, the population were chosen by following these criteria: complete information on BP and at least two valid records of BP. Finally, a total of 14,080 participants were included to confirm our hypothesis.

\section{Ambient $\mathrm{PM}_{2.5}$ Concentrations}

Distribution of $\mathrm{PM}_{2.5}$ were estimated using a hindcast approach based on a two-stage machine learning model. This approach integrated the data of historical emissions and satellite remote sensing measurements. This yielded daily $\mathrm{PM}_{2.5}$ concentrations across a regular $0.1 \times 0.1^{\circ}$ grid over China, from 2000 to 2016. The detailed description of the estimate method has been published previously, with the validation results that the generated concentrations were highly correlated with the ground observations at the monthly $\left(R^{2}=0.71\right)$ and annual $\left(R^{2}=0.77\right)$ scales (Xue et al., 2017). The home address of CHARLS participants can be obtained only at the city level for reasons of confidentiality. Therefore, the $\mathrm{PM}_{2.5}$ concentration data were first converted into city-level and monthly averages, and then linked to the CHARLS respondents according to their spatiotemporal coordinates. The average $\mathrm{PM}_{2.5}$ concentrations in 1 and 2 years before the visiting day were calculated as the long-term exposure value and denoted as $F P M_{1 Y}$ and $F P M_{2 Y}$, respectively. 


\section{Statistics Analyses}

We used the median, interquartile range (IQR), or standard deviation to describe the distributions. To explore the changes in BP before and after the policy intervention, we summarized the population level mean values between the CHARLS waves. Linear mixed-effects regression models with random effects were employed to investigate the associations between ambient $\mathrm{PM}_{2.5}$ concentration and BP. The random terms were used to control for two clustering effects in individual, and community levels. We incorporated a spline term with three degrees of freedom into the regressions to describe the nonlinear effects of ambient temperature. We estimated the effects of a $10 \mu \mathrm{g} / \mathrm{m}^{3}$ decrease in ambient $\mathrm{PM}_{2.5}$ concentration on BP. To examine the robustness of the association between ambient $\mathrm{PM}_{2.5}$ and BP (SBP and DBP), we controlled different groups of confounders resulting in the following four different models:

(I) Model I: $\mathrm{BP}=\beta \times \mathrm{PM}_{2.5}+\beta_{1} \times \mathrm{CF}_{1}+\beta_{2} \times \mathrm{CF}_{2}+\mathrm{Y}_{1}(\mathrm{~S})+\mathrm{\gamma}_{2}(\mathrm{H})$. This model incorporated fixed terms with the $\beta$ coefficients of $\mathrm{PM}_{2.5}, \beta_{1} \beta_{2}$ of potential confounders ( $\mathrm{CF}_{1-2}$ : city and medication), as well as a random intercept for each subject $\gamma_{1}(S)$ and each household $\gamma_{2}(H)$. We controlled for medication status and city location. The participants who were taking anti-hypertensive medicines or had ever taken them during the visiting period were classified into those taking medicines.

(II) Model II: $\mathrm{BP}=\beta \times \mathrm{PM}_{2.5}+\beta_{1-4} \times \mathrm{CF}_{1-4}+\mathrm{Y}_{1}(\mathrm{~S})+\mathrm{Y}_{2}(\mathrm{H})$. This model incorporated fixed terms with the $\beta$ coefficients of $\mathrm{PM}_{2.5}, \beta_{1-4}$ of potential confounders ( $\mathrm{CF}_{1-4}$ : city, medication, temperature, and age), as well as a random intercept for each subject $\gamma_{1}(S)$ and each household $\gamma_{2}(H)$. We further controlled for annual mean temperature, as some studies have reported an inverse association between temperature and BP (Wang et al., 2017). We used natural cubic splines with three knots to account for seasonal temperature variations.

(III) Model III: $\mathrm{BP}=\beta \times \mathrm{PM}_{2.5}+\beta_{1-10} \times \mathrm{CF}_{1-10}+\mathrm{Y}_{1}(\mathrm{~S})+\mathrm{\gamma}_{2}(\mathrm{H})$. This model incorporated fixed terms with the $\beta$ coefficients of $\mathrm{PM}_{2.5}, \beta_{1-10}$ of potential confounders ( $\mathrm{CF}_{1-10}$ : $\mathrm{CF}_{1-4}$, residence, gender, education, marriage, smoking, and drinking alcohol), as well as a random intercept for each subject $\gamma_{1}(S)$ and each household $\gamma_{2}(H)$. We further controlled for the following variables as potential confounders based on previous studies (Chan et al., 2015a; Chen et al., 2015a): gender, education level (below elementary, middle, and above middle), marital status (married and living together or not), residence (urban or rural), smoking (current smoking or not), and drinking alcohol (frequent, rare, never).

(IV)Model IV: $\mathrm{BP}=\beta \times \mathrm{PM}_{2.5}+\beta_{1-16} \times \mathrm{CF}_{1-16}+\mathrm{Y}_{1}(\mathrm{~S})+\mathrm{Y}_{2}(\mathrm{H})$. This model incorporated fixed terms with $\beta$ coefficients of $\mathrm{PM}_{2.5}, \beta_{1-16}$ of potential confounders $\left(\mathrm{CF}_{1-16}\right.$ : $\mathrm{CF}_{1-10}$, temperature maintenance of household, heating fuel, living in a multi-story building or not, renting a house, untidiness of the household, and telephone usage), as well as a random intercept for each subject $Y_{1}(S)$ and each household $\mathrm{Y}_{2}(\mathrm{H})$. We used the same covariates for SBP and DBP.

We conducted a stratification analysis to examine whether the association between ambient $\mathrm{PM}_{2.5}$ and BP was modified by the following factors: age, gender, education level, residence, marital status, smoking, alcohol drinking, and taking medicine. The statistical significance of the effect modification was tested 
by analysis of variance between the Model III and the modified model. The P-value was adjusted by the false discovery rate (FDR) method, which is a way to allow inference when diverse tests are being conducted. Compared with Bonferroni multiple testing method, FDR corrected the P-value in a milder way by means of controlling the proportion of false/true positives to a certain range. The above analysis assumed that BP and explanatory variables showed a linear correlation. To verify this, a generalized additive mixed model with a random intercept was established to explore exposure-response relationship between the ambient $\mathrm{PM}_{2.5}$ and $\mathrm{BP}$ by replacing the linear term of $\mathrm{PM}_{2.5}$ with a set of penalized spline functions:

$\mathrm{BP} \sim \mathrm{g}\left(P M_{2.5}\right)+\beta_{1-10} \times \mathrm{CF}_{1-10}+\mathrm{Y}_{1}(\mathrm{~S})+\mathrm{\gamma}_{2}(\mathrm{H})$

where $\mathrm{g}$ is the smoothing spline term. All statistical analyses were conducted in $\mathrm{R}$ (version 3.5.3; The $\mathrm{R}$ Foundation for Statistical Computing, Vienna Austria). We used two-sided statistical tests, and a $P$-value $<0.05$ was considered significant.

\section{Results}

\section{Population Characteristics}

Their general characteristics of the included 14,080 participants for data analysis are summarized in Table 1. Approximately, $65 \%$ of the participants were from rural area and half of them were female. The proportions below the elementary and elementary and middle education levels were similar between the three visits. Overall, $10 \%$ of them had education level above a middle education. More than $80 \%$ were married and lived together. It revealed that most participants did not frequently have tobacco smoking or drink alcohol. Most of the participants lived in their own houses and more than half lived in a multi-story building with a telephone facility, moderate household temperature, and moderate untidiness. Less than $30 \%$ of the participants took anti-hypertensive drugs. For the mean (IQR) SBP and DBP, there was no significant changes in the CHARLS 2011, 2013, and 2015; for $\mathrm{PM}_{2.5}$ it was decreased significantly from 61.3 (27.6) to $52.8(24.0) \mu \mathrm{g} / \mathrm{m}^{3}$ during 2011-2015. 
Table 1

Characteristics of the study participants

\begin{tabular}{|c|c|c|c|c|}
\hline Characteristic & Visit 1 & Visit 2 & Visit 3 & Overall \\
\hline Number of subjects ${ }^{a}$ & 12,725 & 13,630 & 13,432 & 14,080 \\
\hline Age (years) ${ }^{b}$ & $59.8(14.0)$ & $61.4(15.0)$ & $63.2(14.0)$ & $61.5(14.0)$ \\
\hline \multicolumn{5}{|l|}{ Residence $^{\mathrm{c}}$} \\
\hline Rural & $8288(65)$ & $8866(65)$ & $8810(66)$ & $25964(65)$ \\
\hline Urban & $4437(35)$ & $4764(35)$ & $4622(34)$ & $13823(35)$ \\
\hline \multicolumn{5}{|l|}{ Gender ${ }^{c}$} \\
\hline Female & $6839(54)$ & $7333(54)$ & $7239(54)$ & $21411(54)$ \\
\hline Male & $5885(46)$ & $6297(46)$ & $6192(46)$ & $18374(46)$ \\
\hline Unknown & $1(0)$ & $0(0)$ & $1(0)$ & $2(0)$ \\
\hline \multicolumn{5}{|l|}{ Education $^{\mathrm{c}}$} \\
\hline Below elementary & $6049(48)$ & $5849(43)$ & $5759(43)$ & $17657(44)$ \\
\hline Elementary \& middle & $5357(42)$ & $5163(38)$ & $5118(38)$ & $15638(39)$ \\
\hline Above middle & $1314(10)$ & $1278(9)$ & $1215(9)$ & $3807(10)$ \\
\hline Unknown & $5(0)$ & $1340(10)$ & $1340(10)$ & $2685(7)$ \\
\hline \multicolumn{5}{|c|}{ Married and lived together ${ }^{c}$} \\
\hline No & $2142(17)$ & $2394(18)$ & $2567(19)$ & $7103(18)$ \\
\hline Yes & $10583(83)$ & $11230(82)$ & $10853(81)$ & $32666(82)$ \\
\hline Unknown & $0(0)$ & $6(0)$ & $12(0)$ & $18(0)$ \\
\hline \multicolumn{5}{|l|}{ Smoking ${ }^{c}$} \\
\hline No & $8988(71)$ & $9479(70)$ & 9319 (69) & $27786(70)$ \\
\hline \multirow[t]{2}{*}{ Yes } & $3730(29)$ & $4132(30)$ & $4105(31)$ & $11967(30)$ \\
\hline & $7(0)$ & $19(0)$ & $8(0)$ & $34(0)$ \\
\hline \multicolumn{5}{|l|}{ Drinking alcohol ${ }^{\mathrm{C}}$} \\
\hline Frequent & $3155(25)$ & $3501(26)$ & $3377(25)$ & $10033(25)$ \\
\hline Never & 8564 (67) & $9061(66)$ & 8941 (67) & $26566(67)$ \\
\hline
\end{tabular}




\begin{tabular}{|c|c|c|c|c|}
\hline Characteristic & Visit 1 & Visit 2 & Visit 3 & Overall \\
\hline Rare & $990(8)$ & $1020(7)$ & $1087(8)$ & 3097 (8) \\
\hline Unknown & $16(0)$ & $48(0)$ & $27(0)$ & $91(0)$ \\
\hline \multicolumn{5}{|l|}{ Heating fuel ${ }^{\mathrm{c}}$} \\
\hline Biomass & $3713(29)$ & 3632 (27) & 2011 (15) & $9356(24)$ \\
\hline Central & $912(7)$ & $1360(10)$ & $1358(10)$ & $3630(9)$ \\
\hline Clean & $2226(17)$ & $2762(20)$ & $977(7)$ & $5965(15)$ \\
\hline Coal & $3583(28)$ & $3591(26)$ & $1968(15)$ & $9142(23)$ \\
\hline Unknown & $2291(18)$ & $2285(17)$ & $7118(53)$ & $11694(29)$ \\
\hline \multicolumn{5}{|c|}{ Multi-story building ${ }^{c}$} \\
\hline No & 8235 (65) & 8617 (63) & 7147 (53) & $23999(60)$ \\
\hline Yes & $4442(35)$ & $4958(36)$ & $6197(46)$ & $15597(39)$ \\
\hline Unknown & $48(0)$ & $55(0)$ & $88(1)$ & $191(0)$ \\
\hline \multicolumn{5}{|c|}{ Have telephone ${ }^{c}$} \\
\hline No & $6460(51)$ & $8132(60)$ & $9572(71)$ & $24164(61)$ \\
\hline Yes & $6219(49)$ & $5451(40)$ & $3838(29)$ & $15508(39)$ \\
\hline Unknown & $46(0)$ & $47(0)$ & $22(0)$ & $115(0)$ \\
\hline \multicolumn{5}{|c|}{ Untidiness degree of household ${ }^{c}$} \\
\hline Excellent & $989(8)$ & $1065(8)$ & $1197(9)$ & $3251(8)$ \\
\hline Very clear & $2464(19)$ & $2935(22)$ & $2938(22)$ & $8337(21)$ \\
\hline Clear & $5012(39)$ & $4439(33)$ & $4415(33)$ & $13866(35)$ \\
\hline Fair & $3387(27)$ & $3883(28)$ & $3594(27)$ & $10864(27)$ \\
\hline Poor & $810(6)$ & $972(7)$ & $833(6)$ & $2615(7)$ \\
\hline Unknown & $63(0)$ & $336(2)$ & $455(3)$ & $854(2)$ \\
\hline \multicolumn{5}{|c|}{ Temperature maintenance of household ${ }^{c}$} \\
\hline Very hot & $257(2)$ & $72(1)$ & $74(1)$ & $403(1)$ \\
\hline Hot & $1333(10)$ & $1071(8)$ & $1121(8)$ & $3525(9)$ \\
\hline Bearable & $10615(83)$ & $11625(85)$ & $11387(85)$ & $33627(85)$ \\
\hline
\end{tabular}




\begin{tabular}{|c|c|c|c|c|}
\hline Characteristic & Visit 1 & Visit 2 & Visit 3 & Overall \\
\hline Cold & $397(3)$ & $487(4)$ & $376(3)$ & $1260(3)$ \\
\hline Very cold & $59(0)$ & $23(0)$ & $12(0)$ & $94(0)$ \\
\hline Unknown & $64(1)$ & $352(3)$ & $462(3)$ & $878(2)$ \\
\hline \multicolumn{5}{|l|}{ Renting a house ${ }^{c}$} \\
\hline No & $12402(97)$ & $12887(95)$ & $12655(94)$ & $37944(95)$ \\
\hline Yes & $250(2)$ & $378(3)$ & $367(3)$ & $995(3)$ \\
\hline Unknown & $73(1)$ & $365(3)$ & $410(3)$ & $848(2)$ \\
\hline \multicolumn{5}{|l|}{ Medication ${ }^{\mathrm{C}}$} \\
\hline No & $9661(76)$ & $9685(71)$ & 9014 (67) & $28360(71)$ \\
\hline Yes & 3064 (24) & 3945 (29) & $4418(33)$ & $11427(29)$ \\
\hline $\mathrm{PM}_{2.5} \mathrm{~d}, \mathrm{~b}$ & $61.3(27.6)$ & $59.9(32.5)$ & $52.8(24.0)$ & $58.0(30.4)$ \\
\hline Temperature $^{\mathrm{e}, \mathrm{b}}$ & $13.9(5.0)$ & $14.1(5.8)$ & $14.7(4.4)$ & $14.3(5.0)$ \\
\hline$S B P^{f, b}$ & $130.2(27.7)$ & $130.7(27.7)$ & $129.1(27.3)$ & $130.0(27.3)$ \\
\hline DBP g, b & $75.7(16.0)$ & $76.7(16.0)$ & $75.5(15.3)$ & $76.0(16.0)$ \\
\hline \multicolumn{5}{|l|}{ a Unit: person; } \\
\hline \multicolumn{5}{|c|}{ b The data were described using Mean (IQR ) } \\
\hline \multicolumn{5}{|c|}{${ }^{\mathrm{c}}$ The data were described using Number (\%) } \\
\hline \multicolumn{5}{|c|}{${ }^{\mathrm{d}}$ Average concentration of $\mathrm{PM}_{2.5}$ one year prior to visit time; unit: $\mu \mathrm{g} / \mathrm{m}^{3}$; } \\
\hline \multicolumn{5}{|c|}{${ }^{\mathrm{e}}$ Average temperature one year prior to visit time, unit: ${ }^{\circ} \mathrm{C}$; } \\
\hline \multicolumn{5}{|c|}{ f Systolic blood pressure, unit: mmHg; } \\
\hline 9 Diastolic blood & e, unit: $\mathrm{mmH}$ & & & \\
\hline
\end{tabular}


Table 2

Associations between ambient $\mathrm{PM}_{2.5}$ concentration (Conc.) and blood pressure using the model III.

\begin{tabular}{|c|c|c|}
\hline \multirow[t]{2}{*}{ PM Conc. ${ }^{a}$} & \multicolumn{2}{|c|}{$\beta$ (95\% confidence interval) $^{b}$} \\
\hline & Systolic blood pressure & Diastolic blood pressure \\
\hline $\mathrm{FPM}_{1 Y}$ & $1.24(0.84,1.64)$ & $0.50(0.25,0.75)$ \\
\hline $\mathrm{FPM}_{2 Y}$ & $1.52(0.93,2.11)$ & $0.83(0.46,1.21)$ \\
\hline \multicolumn{3}{|c|}{$\begin{array}{l}\text { average fine particulate matter (FPM) concentration over the past time period before the survey; } \\
\mathrm{FPM}_{1 Y} \text { : average } \mathrm{PM}_{2.5} \text { concentration in the past one year; } \mathrm{FPM}_{2 \mathrm{Y}} \text { : average } \mathrm{PM}_{2.5} \text { concentration in the } \\
\text { past two years; }\end{array}$} \\
\hline
\end{tabular}

The distributions of these values are shown in (Figure 1). The levels of mean $\mathrm{PM}_{2.5}$ decreased significantly during the three visits, SBP and DBP increased at the beginning and then decreased. The overall mean (IQR) levels of $\mathrm{PM}_{2.5}$ during the previous one year of the survey were $58.0(30.4) \mu \mathrm{g} / \mathrm{m}^{3}$. The overall mean (IQR) levels of SBP and DBP were 130.0 (27.3) mmHg and 76.0 (16.0) mmHg, respectively. In addition, SBP generally increased with age, and DBP increased at the beginning and then decreased with age (Figure 1). Although the curves in different years were similar, that derived from the CHARLS 2015 (i.e., the wave after the clean air actions) was lower for most age groups. Because the curves were derived from cross-sectional information without adjustment for confounders, they were used to display the data and do not show the variation in BP with age among Chinese adults.

\section{Association between $\mathrm{PM}_{2.5}$ Exposure and $\mathrm{BP}$}

We explored the associations between ambient $\mathrm{PM}_{2.5}$ concentrations and BP during two different exposure periods using four linear mixed models. It revealed that each reduction of $10 \mu \mathrm{g} / \mathrm{m}^{3} \mathrm{FPM}_{1 \mathrm{Y}}$ was associated with decreases of 1.24 (95\% confidence interval [Cl]: 0.84-1.64) mmHg SBP and 0.50 (95\% Cl: 0.25-0.75) mmHg DBP using model III. Similarly, there were decreases of 1.52 (95\% Cl: 0.93-2.11)

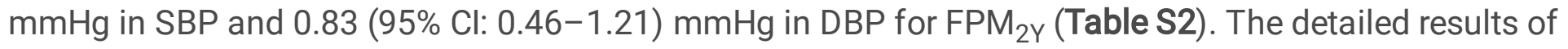
the other models are provided in Table $\mathbf{S 1}(\mathrm{SI})$. Each reduction of $10 \mu \mathrm{g} / \mathrm{m}^{3} \mathrm{FPM}_{1 \mathrm{Y}}$ was associated with decreases of 0.87 (95\% Cl: $0.58-1.16) \mathrm{mmHg}$ SBP and 0.35 (95\% Cl: $0.17-0.54) \mathrm{mmHg}$ DBP using Model I, 1.27 (95\% Cl: 0.87-1.67) mmHg SBP and 0.51 (95\% Cl: 0.26-0.77) mmHg DBP using Model II, and 1.18 (95\% Cl: $0.78-1.58) \mathrm{mmHg}$ SBP and 0.51 (95\% Cl: $0.26-0.77)$ mmHg DBP using Model IV. Overall, all four models indicated that $\mathrm{FPM}_{1 \mathrm{Y}}$ and $\mathrm{FPM}_{2 \mathrm{Y}}$ were positively associated with SBP and DBP.

We further adopted the generalized additive mixed model to evaluate the relative BP changes at different $\mathrm{FPM}_{1 Y}$ concentrations referring to the BP at an $\mathrm{FPM}_{1 Y}$ concentration of $35 \mu \mathrm{g} / \mathrm{m}^{3}$. SBP increased nonlinearly with the increase of $\mathrm{FPM}_{1 \mathrm{Y}}$ concentration, but there was an approximate linear range when the 
$F M_{1 Y}$ concentration was $<70 \mu \mathrm{g} / \mathrm{m}^{3}$ (Figure 2A). DBP increased approximately linearly with the increase of $\mathrm{FPM}_{1 Y}$ without an obvious peak (Figure $2 \mathrm{~B}$ ). The slope of the regression curve of SBP with increasing $\mathrm{FPM}_{1 \mathrm{Y}}$ was larger than that of DBP.

\section{Stratified Analysis}

A stratified analysis was performed to investigate the association between $\mathrm{FPM}_{1 \mathrm{Y}}$ and BP under different levels or grades of various confounders (i.e., medication, age, residence, gender, marriage, smoking, drinking alcohol, and education). The estimated associations between $\mathrm{PM}_{2.5}$ and BP did not vary significantly on inclusion of most of them. For DBP, the association between $\mathrm{FPM}_{1 Y}$ and DBP was greater in the urban residents than in the rural residents without adjusting P-value by the FDR method (Figure 3). Also, a greater association was found in married participants than in single participants. These results suggest that residence and marital status may modify the association between $\mathrm{FPM}_{1 \mathrm{Y}}$ and DBP. However, no significant modification effects of various confounders were found in the association between $F M_{1 Y}$ and DBP when adjusting P-value by the FDR method. For SBP, the association between $\mathrm{FPM}_{1 \mathrm{Y}}$ and SBP was greater in female than in male without adjusting by the FDR method (Figure 3). Similar to DBP, no significant modification effects of various confounders were found when adjusting P-value by the FDR method. The details of the results are provided in Table $\mathbf{S 2}$ (SI).

\section{Discussion}

In this study, we investigated the effect of $\mathrm{PM}_{2.5}$ exposure on $\mathrm{BP}$ using the on-going large population follow-up program in China. The estimated association remained robust after adjusting for a wide range of confounders. Nonlinear regression verified that the decreasing trend in BP with decreases in $\mathrm{PM}_{2.5}$ was almost linear. However, there was a threshold at about $70 \mu \mathrm{g} / \mathrm{m}^{3}$ for the effect of $\mathrm{FPM}_{1 Y}$ on SBP. Overall, our study supports the hypothesis that reducing $\mathrm{PM}_{2.5}$ was significantly associated with a decrease of BP.

Several cross-sectional studies have been conducted to examine the relationship between $\mathrm{PM}_{2.5}$ exposure and BP. Li et al. reported a positive association of long-term exposure to air pollution with both SBP and DBP using a cross-sectional study of 39,207 participants in Henan Province, China (Li et al., 2020). Xie et al. observed increases of 0.569 (95\% Cl: $0.564-0.573) \mathrm{mmHg}$ in SBP and 0.384 (95\% Cl: $0.381-0.388$ ) $\mathrm{mmHg}$ in DBP with $10 \mu \mathrm{g} / \mathrm{m}^{3}$ increase in $\mathrm{PM}_{2.5}$ above $47.9 \mu \mathrm{g} / \mathrm{m}^{3}$, which was conducted among 39 million people across 2,790 counties of 31 provinces in China (Xie et al., 2018b). These previous crosssectional studies provide valuable evidence about the relationship between $\mathrm{PM}_{2.5}$ and $\mathrm{BP}$. In addition, some cohort studies have also been conducted to examine this relationship. For example, Zhang et al. reported a positive associations of $\mathrm{PM}_{2.5}$ exposure with both SBP and DBP in their cohort study of 361,560 participants in Taiwan (Zhang et al., 2018). Adar et al. reported decreases in pollution and BP over time among 5,527 older adults in a long-term follow-up prospective cohort (Adar et al., 2018). Overall, these cohort or cross-sectional studies provide certain evidences about the positive associations 
between $\mathrm{PM}_{2.5}$ and $\mathrm{BP}$. Overall, the findings in our study were consistent with those in the previous reports. However, our study provided more valid evidence in consideration of the study method and exposure scenario. It has been well known that repeated-measurement studies have a stronger ability to verify causality than cross-sectional studies, which has been widely used as a special study design in environmental epidemiology. However, it is difficult to conduct such studies on large-scale populations due to the high cost and requirement for frequent visits. The sample sizes of previous repeatedmeasurement studies on the relationship between $\mathrm{PM}_{2.5}$ and $\mathrm{BP}$ were less than several hundreds of participants to the best of our knowledge (Mu et al., 2014; Huang et al., 2018b; Santos et al., 2019). Our repeated-measurement study from the CHARLS survey including a large sample size of 14,080 participants with at least two valid visits had a great advantage.

Likewise, we chose the exposure scenario of these population with the historically strict enforcement of air pollution prevention and control plan in China. Such quasi-experimental study provides a unique chance to test our hypothesis. Many countries have mitigated air pollution in past decades and the benefits of reducing the population prevalence of hypertension and other outcomes have been documented (Laden et al., 2006; Pope et al., 2009; Bo et al., 2019b). For example, a previous study observed that reducing long-term $\mathrm{PM}_{2.5}$ is associated with a lower risk of hypertension when air pollution is considerably mitigated among adults in Taiwan during 2001-2014 (Bo et al., 2019a). Huang et al. discovered the potential benefits of air pollution control in urban China by assuming different air quality improvement scenarios. They reported that a mean $\mathrm{PM}_{2.5}$ reduction to Beijing Olympic levels by 2030 would gain about 241,000 (95\% uncertainty interval, 189,000-293,000) life-years annually (Huang et al., 2017). Wang et al. estimated the $\mathrm{PM}_{2.5}$-associated disease burden using models of virtual scenarios and reported that improving air pollution would reduce the number of $\mathrm{PM}_{2.5}$-related premature deaths in China (Wang et al., 2019b). Our study examined the benefits of improving air quality on BP with a stronger causal association using a natural scenario of policy-driven air quality improvement in China.

We also adopted a nonlinear regression model to verify that BP increased linearly with the increase of $\mathrm{FPM}_{1 Y}$ when the $\mathrm{FPM}_{1 Y}$ concentration was $<70 \mu \mathrm{g} / \mathrm{m}^{3}$. Notably, a threshold was observed for the relationship between $\mathrm{FPM}_{1 Y}$ and SBP. By contrast, a repeated-measures study conducted in China reported that both SBP and DBP increase linearly with $\mathrm{PM}_{2.5}$ when its concentration is $<50 \mu \mathrm{g} / \mathrm{m}^{3}$. There is a threshold in the dose-response curve between PM $_{2.5}$ and SBP, as well as DBP (Chang et al., 2015). Fan et al. reported a "J" shaped concentration-response curve for the relationship between $\mathrm{PM}_{2.5}$ and SBP using a generalized additive mixed model. They observed remarkable increases in SBP when $\mathrm{PM}_{2.5}$ concentrations were $>100 \mu \mathrm{g} / \mathrm{m}^{3}$, whereas no significant changes in SBP were observed at $\mathrm{PM}_{2.5}$ concentrations $<100 \mu \mathrm{g} / \mathrm{m}^{3}$ (Fan et al., 2019). A cross-sectional study of 4,121 elderly people conducted in the United States analyzed the dose-response curve between $\mathrm{PM}_{2.5}$ and SBP. They found that SBP increases approximately linearly with the increase of $\mathrm{PM}_{2.5}$ without an obvious threshold (Honda et al., 2018), which was possibly due to the relatively lower average $\mathrm{PM}_{2.5}$ concentration of $10.4 \mu \mathrm{g} / \mathrm{m}^{3}$. Overall, 
the thresholds could not be determined in our study, which may due to a relatively lower average FPM $1 Y$ of $58.0 \mu \mathrm{g} / \mathrm{m}^{3}$.

Without adjusting the statistical results by FDR method, the effect of $\mathrm{PM}_{2.5}$ on DBP was stronger in urban participants than rural participants. Also, the effect of $\mathrm{PM}_{2.5}$ on DBP seemed to be stronger in the married population than the single population. The similar phenomenon was ever observed in a nationwide crosssectional study of among 17,708 participants in China. They reported the effects of $\mathrm{PM}_{2.5}$ on hypertension prevalence were stronger among middle-aged, obese and urban participants (Liu et al., 2017). One possible reason is that the $\mathrm{PM}_{2.5}$ composition in rural and urban areas is different. For example, automobile exhaust and coal combustion generally as contributions in urban areas (Wu et al., 2015), while the residential solid fuel burning was an important source. Meng et al. summarized the contributions of nonresidential and residential activities to ambient $\mathrm{PM}_{2.5}$ concentrations. They reported that for rural areas where $\mathrm{PM}_{2.5}$ levels in the indoor air $\left(95 \pm 34 \mu \mathrm{g} / \mathrm{m}^{3}\right)$ were significantly higher than those in urban indoor air $\left(58 \pm 23 \mu \mathrm{g} / \mathrm{m}^{3}\right.$ ) because of the strong dependence of rural residents on solid fuels. In contrast, ambient $\mathrm{PM}_{2.5}$, which is mainly from nonresidential sources, was higher in urban (45 \pm $19 \mu \mathrm{g} / \mathrm{m}^{3}$ ) than in rural areas $\left(22 \pm 15 \mu \mathrm{g} / \mathrm{m}^{3}\right.$ ) (Meng et al., 2019). Interestingly, the married population was more sensitive to the adverse effects of $\mathrm{PM}_{2.5}$ on BP. Previous studies reported that married and single people have different economic conditions, living habits, and psychosocial stress (Hicken et al., 2014; Weaver et al., 2019). Li et al. determined that the effect of $\mathrm{PM}_{2.5}$ on BP can be modified by behavioral factors, including tobacco smoking, drinking alcohol, high-fat diet, and frequent physical activity (Zhang et al., 2018; Li et al., 2019). In our study, the average age, as well as the proportions of participants with high frequencies of tobacco smoking and drinking alcohol, were larger in single participants than those in married participants. In other words, age and living habits (smoking and drinking alcohol) may play a more important role in single participants than in married participants. It suggested that the BP of married population may be more sensitive to the $\mathrm{PM}_{2.5}$ exposure. However, these results cannot be well explained using the current data and more evidence from additional studies are still needed.

Our study has two important limitations. First, the $\mathrm{PM}_{2.5}$ exposure assessment was based on historical estimates; we did not conduct exact personal exposure measurements, nor did we have information on indoor air quality. This uncertainty in the $\mathrm{PM}_{2.5}$ concentration could lead to exposure misclassifications and bias the results. Similarly, coarseness in the exposure assessment due to the lack of addresses could also lead to exposure misclassification, despite that previous studies used a similar method, e.g., a six U.S. cities prospective cohort study measured air-pollution data in each community at a centrally located air-monitoring station (Dockery et al., 1993). Second, the underlying mechanisms for the modifying effects of population residence and marital status cannot be well explained using the current information. However, to the best of our knowledge, our study examined the largest population to investigate the effect of $\mathrm{PM}_{2.5}$ on $\mathrm{BP}$ using the repeated-measurement study design conducted in China. 
Particularly, our study results provided the direct evidences on the protective effects of the improved air quality on the blood pressure. Above all, our conclusion warrants further studies for confirmation.

\section{Conclusions}

We concluded that reducing long-term $\mathrm{PM}_{2.5}$ exposure could decrease BP among middle-aged and elderly residents in China. Our findings provide an important perspective of improve the cardiovascular health from the air pollution control. It will provide a reference for related air quality improvement.

\section{Declarations}

Funding: None

ACKNOWLEDGEMENTS: We would like to express our gratitude to the suggestions the researchers from the working group of environmental exposure and human health of the China Cohort Consortium (http://chinacohort.bjmu.edu.cn/).

Disclosures: None

\section{References}

1. Adar et al (2018) Longitudinal Analysis of Long-Term Air Pollution Levels and Blood Pressure: A Cautionary Tale from the Multi-Ethnic Study of Atherosclerosis. Environ Health Perspect 126:107003

2. Auchincloss etal (2008) Associations between recent exposure to ambient fine particulate matter and blood pressure in the Multi-ethnic Study of Atherosclerosis (MESA). Environ Health Perspect 116:486-491

3. Bao M, Wang $L$ (2020) The longitudinal trend of hypertension prevalence in Chinese adults from 1959 to 2018: a systematic review and meta-analysis. Annals of Palliative Medicine 9:2485-2497

4. Bilenko et al (2015) Associations between particulate matter composition and childhood blood pressure-The PIAMA study. Environ Int 84:1-6

5. Bo et al (2019a) Dynamic Changes in Long-Term Exposure to Ambient Particulate Matter and Incidence of Hypertension in Adults. 74:669-677

6. Bo et al (2019b) Dynamic Changes in Long-Term Exposure to Ambient Particulate Matter and Incidence of Hypertension in Adults. Hypertension 74:669-677

7. Bochud et al (2012) Gene-environment interactions of selected pharmacogenes in arterial hypertension. Expert Rev Clin Pharmacol 5:677-686

8. Chan et al (2015a) Long-term air pollution exposure and blood pressure in the sister study. Environ Health Perspect 123:951-958

9. Chan et al (2015b) Long-Term Air Pollution Exposure and Blood Pressure in the Sister Study. Environ Health Perspect 123:951-958 
10. Chang et al (2015) Short-term exposure to noise, fine particulate matter and nitrogen oxides on ambulatory blood pressure: A repeated-measure study. Environ Res 140:634-640

11. Chen et al (2015a) Associations between long-term air pollutant exposures and blood pressure in elderly residents of Taipei city: A cross-sectional study. Environ Health Perspect 123:779-784

12. Chen et al (2015b) Associations between Long-Term Air Pollutant Exposures and Blood Pressure in Elderly Residents of Taipei City: A Cross-Sectional Study. Environ Health Perspect 123:779-784

13. Chuang et al (2011) Long-term air pollution exposure and risk factors for cardiovascular diseases among the elderly in Taiwan. Occup Environ Med 68:64-68

14. Chung et al (2015) Association of PNC, BC, and $\mathrm{PM}_{2.5}$ measured at a central monitoring site with blood pressure in a predominantly near highway population. Int J Environ Res Public Health $12: 2765-2780$

15. Curto et al (2019) Ambient Particulate Air Pollution and Blood Pressure in Peri-urban India. Epidemiology 30:492-500

16. Dai et al (2016) Use of the adaptive LASSO method to identify $\mathrm{PM}_{2.5}$ components associated with blood pressure in elderly men: The veterans affairs normative aging study. Environ Health Perspect $124: 120-125$

17. Desai AN (2020) High Blood Pressure Jama 324:1254-1255

18. Dockery et al (1993) An Association between Air Pollution and Mortality in Six U.S. Cities. N Engl J Med 329:1753-1759

19. Dvonch et al (2009) Acute Effects of Ambient Particulate Matter on Blood Pressure. 53:853-859

20. Fan etal (2019) Acute Effects of High-Level PM $_{2.5}$ Exposure on Central Blood Pressure. Hypertension 74:1349-1356

21. Feng etal (2019) Defending blue sky in China: Effectiveness of the "Air Pollution Prevention and Control Action Plan" on air quality improvements from 2013 to 2017. J Environ Manage 252:109603

22. Forouzanfar etal (2017) Global Burden of Hypertension and Systolic Blood Pressure of at Least 110 to $115 \mathrm{~mm} \mathrm{Hg}, 1990-2015$. JAMA 317:165-182

23. Fuks et al (2017) Long-term exposure to ambient air pollution and traffic noise and incident hypertension in seven cohorts of the European study of cohorts for air pollution effects (ESCAPE). Eur Heart J 38:983-990

24. Hay et al (2020) Hypertension and Age-Related Cognitive Impairment: Common Risk Factors and a Role for Precision Aging. Curr Hypertens Rep 22:80

25. Hicken et al (2014) Fine particulate matter air pollution and blood pressure: the modifying role of psychosocial stress. Environ Res 133:195-203

26. Honda et al (2018) Associations of long-term fine particulate matter exposure with prevalent hypertension and increased blood pressure in older Americans. Environ Res 164:1-8

27. Huang et al (2017) Potential Cardiovascular and Total Mortality Benefits of Air Pollution Control in Urban China. Circulation 136:1575-1584 
28. Huang et al (2018a) Health impact of China's Air Pollution Prevention and Control Action Plan: an analysis of national air quality monitoring and mortality data. The Lancet Planetary health 2:313323

29. Huang et al (2019) Long-Term Exposure to Fine Particulate Matter and Hypertension Incidence in China. Hypertension 73:1195-1201

30. Huang et al (2018b) Short-Term Blood Pressure Responses to Ambient Fine Particulate Matter Exposures at the Extremes of Global Air Pollution Concentrations. Am J Hypertens 31:590-599

31. Khera et al (2018) Impact of 2017 ACC/AHA guidelines on prevalence of hypertension and eligibility for antihypertensive treatment in United States and China: nationally representative cross sectional study. BMJ (Clinical research ed 362:k2357

32. Laden et al (2006) Reduction in fine particulate air pollution and mortality: Extended follow-up of the Harvard Six Cities study. Am J Respir Crit Care Med 173:667-672

33. Lewington et al (2016) The Burden of Hypertension and Associated Risk for Cardiovascular Mortality in China. JAMA internal medicine 176:524-532

34. Li etal (2019) Associations of long-term exposure to ambient PM1 with hypertension and blood pressure in rural Chinese population: The Henan rural cohort study. Environ Int 128:95-102

35. Li et al (2020) Associations between long-term exposure to air pollution and blood pressure and effect modifications by behavioral factors. Environ Res 182:109109-109109

36. Lin etal (2017) Long-Term Effects of Ambient $\mathrm{PM}_{2.5}$ on Hypertension and Blood Pressure and Attributable Risk Among Older Chinese Adults. Hypertension 69:806-812

37. Liu et al (2017) Associations between ambient fine particulate air pollution and hypertension: A nationwide cross-sectional study in China. Sci Total Environ 584-585:869-874

38. Ma et al (2021) Trends in hypertension prevalence, awareness, treatment and control rates among Chinese adults, 1991-2015. J Hypertens 39:740-748

39. Meng etal (2019) Energy and air pollution benefits of household fuel policies in northern China. Proc. Natl. Acad. Sci. U. S. A. 116, 16773-16780

40. Minicuci etal (2019) Data Resource Profile: Cross-national and cross-study sociodemographic and health-related harmonized domains from SAGE plus CHARLS, ELSA, HRS, LASI and SHARE (SAGE+ Wave 2). Int J Epidemiol 48:14-14j

41. Mu et al (2014) Peak expiratory flow, breath rate and blood pressure in adults with changes in particulate matter air pollution during the Beijing Olympics: A panel study. Environ Res 133:4-11

42. Pope et al (2009) Fine-Particulate Air Pollution and Life Expectancy in the United States. 360, 376386

43. Rao et al (2018) Effect of Particulate Matter Air Pollution on Cardiovascular Oxidative Stress Pathways. Antioxid Redox Signal 28:797-818

44. Santos et al (2019) Exposure to fine particles increases blood pressure of hypertensive outdoor workers: A panel study. Environ Res 174:88-94 
45. Stanaway etal (2018) Global, regional, and national comparative risk assessment of 84 behavioural, environmental and occupational, and metabolic risks or clusters of risks for 195 countries and territories, 1990-2013;2017: a systematic analysis for the Global Burden of Disease Study 2017. The Lancet 392:1923-1994

46. van Oort et al (2020) Association of Cardiovascular Risk Factors and Lifestyle Behaviors With Hypertension: A Mendelian Randomization Study. Hypertension 76:1971-1979

47. Wang etal (2019a) Spatio-Temporal Variation Characteristics of $\mathrm{PM}_{2.5}$ in the Beijing-Tianjin-Hebei Region, China, from 2013 to 2018.Int J Environ Res Public Health16

48. Wang etal (2017) Environmental ambient temperature and blood pressure in adults: A systematic review and meta-analysis. Sci Total Environ 575:276-286

49. Wang et al (2019b) Estimation of $\mathrm{PM}_{25}$-associated disease burden in China in 2020 and 2030 using population and air quality scenarios: a modelling study. The Lancet Planetary health 3:e71-e80

50. Wang et al (2018) Status of Hypertension in China: Results From the China Hypertension Survey, 2012-2015. Circulation 137:2344-2356

51. Weaver et al (2019) Neighborhood sociodemographic effects on the associations between long-term $\mathrm{PM}_{2.5}$ exposure and cardiovascular outcomes and diabetes. Environmental epidemiology (Philadelphia, Pa.) 3

52. Wu etal (2020) Ambient Airborne Particulates of Diameter $\leq 1 \mu \mathrm{m}$, a Leading Contributor to the Association Between Ambient Airborne Particulates of Diameter $\leq 2.5 \mu \mathrm{m}$ and Children's Blood Pressure. Hypertension 75:347-355

53. Wu etal (2015) Association of chemical constituents and pollution sources of ambient fine particulate air pollution and biomarkers of oxidative stress associated with atherosclerosis: A panel study among young adults in Beijing, China. Chemosphere 135:347-353

54. Xie et al (2018a) Long-Term Effects of Ambient Particulate Matter (With an Aerodynamic Diameter $\leq$ $2.5 \mu \mathrm{m}$ ) on Hypertension and Blood Pressure and Attributable Risk Among Reproductive-Age Adults in China.J Am Heart Assoc7

55. Xie et al (2018b) Long-Term Effects of Ambient Particulate Matter (With an Aerodynamic Diameter $\leq 2.5 \mu \mathrm{m}$ ) on Hypertension and Blood Pressure and Attributable Risk Among Reproductive-Age Adults in China.J Am Heart Assoc7

56. Xue et al (2019a) Rapid improvement of $\mathrm{PM}_{2.5}$ pollution and associated health benefits in China during 2013-2017. Science China Earth Sciences

57. Xue etal (2017) Fusing Observational, Satellite Remote Sensing and Air Quality Model Simulated Data to Estimate Spatiotemporal Variations of $\mathrm{PM}_{2.5}$ Exposure in China.Remote Sensing 9

58. Xue et al (2019b) Spatiotemporal continuous estimates of $\mathrm{PM}_{2.5}$ concentrations in China, 20002016: A machine learning method with inputs from satellites, chemical transport model, and ground observations. Environ Int 123:345-357 
59. Yang et al (2018) Global association between ambient air pollution and blood pressure: A systematic review and meta-analysis. Environmental pollution (Barking, Essex: 1987) 235, 576-588

60. Zhang et al (2019a) Drivers of improved $\mathrm{PM}_{2.5}$ air quality in China from 2013 to 2017. Proc. Natl. Acad. Sci. U. S. A. 116, 24463-24469

61. Zhang et al (2019b) Exposure to ambient particulate matter air pollution, blood pressure and hypertension in children and adolescents: A national cross-sectional study in China. Environ Int 128:103-108

62. Zhang et al (2018) Long-Term Exposure to Fine Particulate Matter, Blood Pressure, and Incident Hypertension in Taiwanese Adults. Environ Health Perspect 126:017008

63. Zhao et al (2014) Cohort profile: the China Health and Retirement Longitudinal Study (CHARLS). Int J Epidemiol 43:61-68

Figures
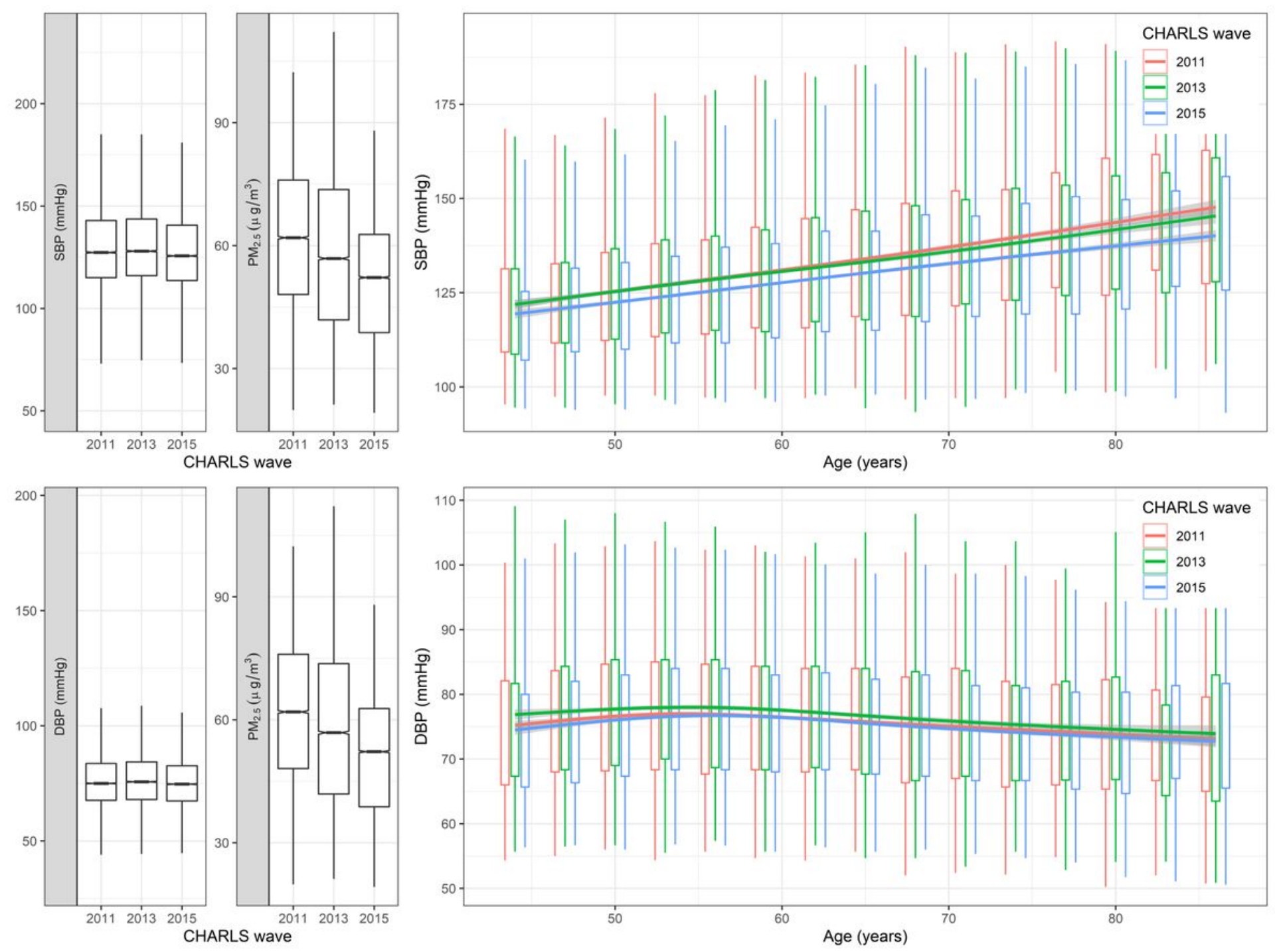

Figure 1 
Changes in PM2.5 and BP. Left panel, distribution of SBP, DBP, and PM2.5 by CHARLS wave; right panel, age-specific distribution of the waves; smoothed curves for BP and age were derived using the spline approach.
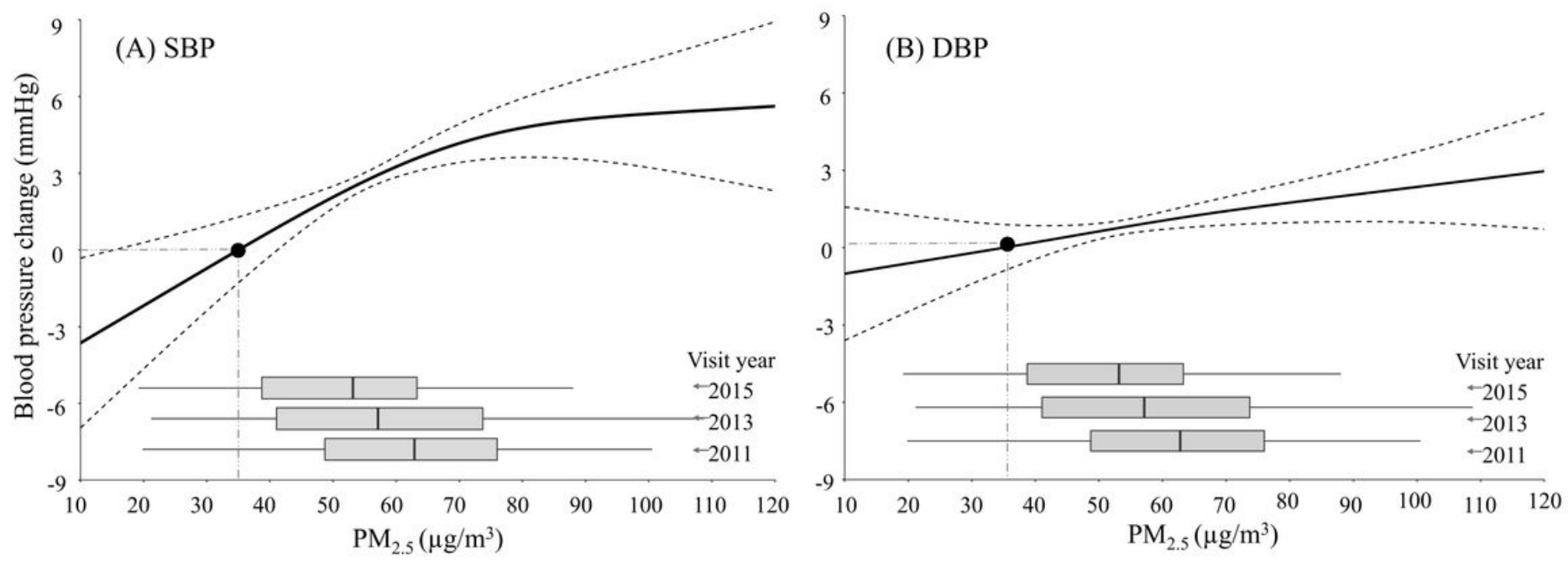

\section{Figure 2}

Non-linear association between average FPM1Y and systolic (A) and diastolic (B) blood pressures. DBP = diastolic blood pressure; PM2.5 = fine particulate matter; SBP = systolic blood pressure. 


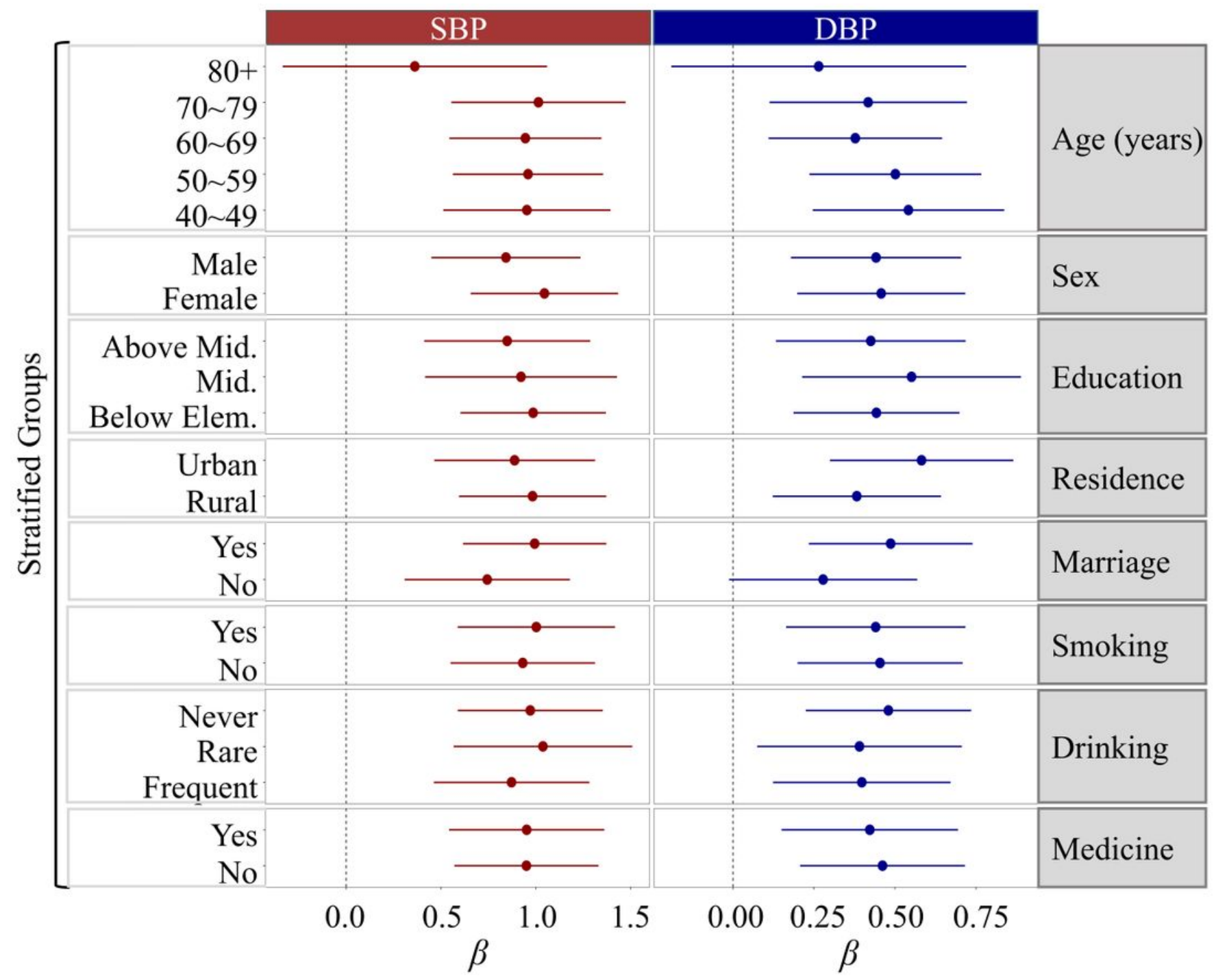

Figure 3

Associations between average PM2.5 concentration over the past one year and blood pressure. DBP = diastolic blood pressure; PM2.5 = fine particulate matter; SBP = systolic blood pressure, Mid. = middle, Elem. $=$ elementary.

\section{Supplementary Files}

This is a list of supplementary files associated with this preprint. Click to download.

- 2SM20211109.docx

- graphicalabstract.tif 